\title{
A Reynolds Stress Closure for Compressible Turbulent Flow
}

\author{
H. Khlifi ${ }^{1 \dagger}$ and T. Lili ${ }^{2}$ \\ ${ }^{1}$ Département de Physique, Faculté des Sciences de Tunis, Campus Universitaire, 1060, Tunis, Tunisie \\ ${ }^{2}$ Département de Physique, Faculté des Sciences de Tunis, Campus Universitaire, 1060, Tunis, Tunisie. \\ $†$ Corresponding Author Email: khlifihachmi@yahoo.fr
}

(Received April 25, 2010; accepted March 13, 2011)

\begin{abstract}
Several studies of compressible flows show that the pressure-strain is the main indicator of the structural compressibility effects. Undoubtedly, this term controls the change in the Reynolds stress anisotropy. Regarding the model of Adumitroiae et al., the slow part of the pressure strain correlation like the Rotta model uses the standard coefficient $\mathrm{C}_{1}$. The model predictions do not show large differences when compressibility increases. Correction of this coefficient using the turbulent Mach number is proposed. The two forms model of Adumitroiae et al. (with and without correction of $C_{1}$ ) are considered to study compressible mixing layers. The obtained results show that the predictions of the proposed compressibility correction model agree with the experiment results of Goebel and Dutton.
\end{abstract}

Keywords: Turbulence flow, Compressible, Pressure-strain, Models of turbulence, Mixing layer.

\section{NOMENCLATURE}

$\begin{array}{ll}a & \text { speed of sound } \\ b_{i j} & \text { Reynolds stress anisotropy } \\ C_{p} & \text { specific heat at constant pressure } \\ C v & \text { specific heat at constant volume } \\ d & \text { fluctuation of the dilatation } \\ K & \text { turbulent kinetic energy } \\ p & \text { pressure } \\ R & \text { specific gas constant } \\ R_{i j} & \text { Reynolds stress } \\ M c & \text { convective Mach number } \\ M_{t} & \text { turbulent Mach number } \\ T & \text { temperature } \\ t & \text { time }\end{array}$

\section{INTRODUCTION}

Compressible turbulence modelling plays an important role in the study of many applications such as aerospace and engineering problems. The techniques and methodologies that have been proved useful in the incompressible flows may represent a reasonable way to develop compressible turbulence models. The direct extension of incompressible models was used in simulating different compressible flows. That one was observed when the standard $(K-\varepsilon)$ and the Reynolds stress closures were extended to compressible flows with an explicit account of compressibility effects, by considering dilatational terms models. These terms which have been recognized as important indicators of compressibility are the pressure-dilatation correlation

$\begin{array}{ll}u_{i} & \text { velocity in the direction, } x_{i} \\ \gamma & \text { specific heat ratio } \\ \varepsilon_{s} & \text { solenoidal part of the dissipation } \\ \mu & \text { viscosity coefficient } \\ \rho & \text { density } \\ \varepsilon & \text { dissipation rate of turbulence } \\ \delta_{i j} & \text { Kronecker delta } \\ \kappa & \text { thermal conductivity } \\ \tau_{i j} & \text { viscous stress tensor } \\ \varepsilon_{C} & \text { dilatational part of the dissipation } \\ ()^{\prime} & \text { Favre fluctuation } \\ ()^{\prime} & \text { Reynolds fluctuation }\end{array}$

and compressible dissipation rate appearing in the turbulent kinetic energy transport equation. It has been shown that this practice of modelling, called compressibility correction models, may be able to reproduce the compressibility phenomenon at small values of Mach number. But, when the compressibility effects are more significant, the extended models do neither predict correctly the decrease in spreading rate of mixing layers, as it is observed in the experiments of Goebel et al. (1991) and Elliot et al. (1990), nor the reduction in the growth rate of turbulent kinetic energy Sarkar (1995). The deficiencies of such closure is due principally to the use of the incompressible models of the pressure strain correlation which controls the level of Reynolds stress anisotropy. However, new models taking into account structural compressibility effects are needed for the pressure strain correlation. The present 
work focuses on this major issue. In this context, Adumitroiae et al. (1999) use the incompressible modelling approach to modify the linear pressure strain. In the present study, recent compressible models for the pressure-dilatation correlation have been used to modify the Rotta model (1951) for the slow part of the pressure-strain correlation. This modification make the standard coefficient $C_{1}$ dependant on the turbulent Mach number. This model is linked to the Adumitroaie model (1999). The resultant model is applied to compressible mixing layers, showing an acceptable success to predict the reduced growth rate and the decrease of the Reynolds stress peaks when the convective Mach number increases.

\section{GoVERNING EQUATIONS}

The general equations governing the motion of compressible fluid are the Navier stokes equations. They can be written as follows for mass, momentum and energy conservation:

$\frac{\partial}{\partial t} \rho+\left(\rho u_{i}\right)_{, i}=0$

$\frac{\partial}{\partial t}\left(\rho u_{i}\right)+\left(\rho u_{i} u_{j}\right)_{, j}=\left(\sigma_{i j}\right)_{, j}$

$\frac{\partial}{\partial t}(\rho e)+\left(\rho e u_{j}\right)_{, j}=\left(\sigma_{i j} u_{j}\right)_{, j}$

$e=c_{v} T+\left(u_{i} u_{i} / 2\right), \sigma_{i j}=-p \delta_{i j}+\tau_{i j}$

The Favre averaged-equations are :

$\frac{\partial}{\partial t} \bar{\rho}+\left(\rho \tilde{u}_{i}\right)_{, i}=0$

$\frac{\partial}{\partial t}\left(\bar{\rho} \tilde{u}_{i}\right)+\left(\rho \tilde{u}_{i} \tilde{u}_{j}\right)_{, j}=-\left(\bar{\rho} R_{i j}\right)_{, j}$

$$
+\left(\bar{\rho} \bar{\tau}_{i j}\right)_{, j}
$$

$\frac{\partial}{\partial t}\left(\bar{\rho} \overline{C_{v}} \tilde{T}\right)+\left(\bar{\rho} \overline{C_{v}} \tilde{T} \tilde{u}_{i}\right)_{, i}=-\pi_{d}+\bar{\Phi}$

$$
+\left(k \tilde{T}_{, i}\right)_{, i}-\overline{Q_{i, i}}
$$

where $R_{i j}=\overline{\rho u_{i}^{\prime \prime} u_{j}^{\prime \prime}} / \bar{\rho}$

$Q_{i}=C_{v} \overline{\rho u_{i}^{\prime \prime} T^{\prime \prime}}$

$\bar{\Phi}=\overline{\tau_{i j} u_{i, j}}-\bar{p} \tilde{u}_{i, i}-\bar{p} \overline{u_{i}^{\prime \prime}}$

$\overline{\tau_{i j}}=\mu\left(\tilde{u}_{i, j}+\tilde{u}_{j, i}\right)-\frac{2}{3} \tilde{u}_{k, k} \delta_{i j}$

$$
\pi_{d}=\overline{p^{\prime} u_{i, i}^{\prime \prime}}=\overline{p^{\prime} d^{\prime}} \quad, \quad d^{\prime}=u_{i, i}^{\prime}
$$

To close the above equations, the Reynolds stress must be solutions of the following transport equations, namely

$$
\begin{array}{r}
\frac{\partial}{\partial t}\left(\bar{\rho} R_{i j}\right)+\left(\bar{\rho} R_{i j} \tilde{u}_{m}\right)_{, m}=P_{i j}+D_{i j} \\
+\phi_{i j}+\varepsilon_{i j}+T_{i j} \\
P_{i j}=-\left(\bar{\rho} R_{i m} \tilde{u}_{j, m}+\bar{\rho} R_{j m} \overline{u_{i, m}}\right)
\end{array}
$$

$$
\begin{aligned}
\varepsilon_{i j}= & -\overline{\tau_{i m}^{\prime \prime} u_{j, m}^{\prime \prime}}-\overline{\tau_{j m}^{\prime \prime} u_{i, m}^{\prime \prime}} \\
D_{i j}= & -\overline{\left(\rho u_{i}^{\prime \prime} u_{j}^{\prime \prime} u_{m}^{\prime \prime}\right.}+\overline{p^{\prime} u_{j}^{\prime \prime}} \delta_{i m} \\
& \left.+\overline{p^{\prime} u_{j}^{\prime}} \delta_{j m}-\overline{\tau_{i m}^{\prime \prime} u_{j}^{\prime \prime}}-\overline{\tau_{j m}^{\prime \prime} u_{i}^{\prime \prime}}\right) \\
\phi_{i j} & =\overline{p^{\prime}\left(u_{i, j}^{\prime \prime}+u_{j, i}^{\prime \prime}\right)} \\
T_{i j}= & -\overline{p_{, j}^{\prime}} \overline{u_{i}^{\prime \prime}}-\overline{p_{, i}} \overline{u_{j}^{\prime \prime}} \\
& +\tilde{\tau}_{i m, m} \overline{u_{j}^{\prime \prime}}+\tilde{\tau}_{j m, m} \overline{u_{i}^{\prime \prime}}
\end{aligned}
$$

\section{Models of Turbulence}

\subsection{Models of the Pressure-Strain Correlation}

*Model of Launder Reece and Rodi(LRR) (1975)

$$
\begin{aligned}
& \phi_{i j}^{*}=C_{1} \bar{\rho} \varepsilon_{s} b_{i j}+\frac{4}{5} \bar{\rho} k\left(\tilde{S}_{i j}-\frac{1}{3} \tilde{S}_{l l} \delta_{i j}\right) \\
& +2 \bar{\rho} k\left(1-C_{3}\right)\left[b_{i m} \tilde{S}_{j m}+b_{j m} \tilde{S}_{i m}\right] \\
& +\bar{\rho} k\left(1-C_{4}\right)\left[b_{i m} \tilde{\Omega}_{j m}+b_{j m} \tilde{\Omega}_{i m}\right]
\end{aligned}
$$

where:

$$
\tilde{S}_{i j}=0.5\left(\tilde{U}_{i, j}+\tilde{U}_{j, i}\right), \tilde{\Omega}_{i j}=0.5\left(\tilde{U}_{i, j}-\tilde{U}_{j, i}\right)
$$

and $b_{i j}=\left(R_{i j} / 2 K-\delta_{i j} / 3\right)$

* Model of Adumitroiae et al. (1999)

Adumitroiae et al. (1990) assumed that incompressible modeling approach of the pressure strain correlation can be used to develop turbulent models taking into account compressibility effects. Considering a none zero divergence for the velocity fluctuation called the compressibility continuity constraint and using different models for the pressure dilatation which is proportional to the trace of the pressure strain, their model for the linear part of this term is written as:

$$
\begin{aligned}
& \phi_{i j}^{*}=C_{1} \bar{\rho} \varepsilon_{s} b_{i j}+\left(\frac{4}{5}+\frac{2}{5} d_{1}\right) \bar{\rho} k\left(\tilde{S}_{i j}-\frac{1}{3} \tilde{S}_{l l} \delta_{i j}\right) \\
& +2 \bar{\rho} k\left(1-C_{3}+2 d_{2}\right)\left[b_{i m} \tilde{S}_{j m}+b_{j m} \tilde{S}_{i m}\right. \\
& \left.-\frac{2}{3} d_{2} b_{n l} \tilde{S}_{n l} \delta_{i j}\right]+\bar{\rho} k\left(1-C_{4}-2 d_{2}\right) \\
& {\left[b_{i m} \tilde{\Omega}_{j m}+b_{j m} \tilde{\Omega}_{i m}-\frac{4}{3} d_{2} b_{i j} \tilde{S}_{n l}\right]}
\end{aligned}
$$

The compressibility coefficients $d_{1}$ and $d_{2}$ are determined on the basis of some compressible closures of the pressure-dilatation correlation.

\subsection{A Modeling for the Slow Part of the Pressure-Strain Correlation}

In this section, the purpose is to investigate the compressibility effect in the slow part of the pressurestrain appearing in the turbulent Reynolds stress transport equation. Classically, for the compressible turbulent flows, the pressure-strain correlation can be divided in two terms as

$\overline{p^{\prime} u_{i, j}^{\prime}}=\left(\overline{p^{\prime} u_{i, j}^{\prime}}\right)_{i n c}+\left(\overline{p^{\prime} u_{i, j}^{\prime}}\right)_{c o m p}$

where $\left(\overline{p^{\prime} u_{i, j}^{\prime}}\right)_{i n c}$ and $\left(\overline{p^{\prime} u_{i, j}^{\prime}}\right)_{c o m p}$ are the incompressible and compressible parts of $\left(\overline{p^{\prime} u_{i, j}^{\prime}}\right)$ respectively.

The trace of these terms are:

$$
\begin{aligned}
& \left(\overline{p^{\prime} u_{i, i}^{\prime}}\right)_{i n c}=0 \\
& \left(\overline{p^{\prime} u_{i, i}^{\prime}}\right)_{\text {comp }}=\overline{p^{\prime} d^{\prime}}
\end{aligned}
$$


It is natural that the first term in the right hand side of Eq. (20) is represented by using the simplest Rotta's model as:

$\left.\overline{\left(p^{\prime} u_{i, j}^{\prime}\right.}\right)_{i n c}=-C_{1} \varepsilon b_{i j}$

Where $C_{1}$ is a constant model assigned to a value of about 3 .

To give a complete model for the total slow part of the pressure-strain correlation, an expression of the compressible term $\left(\overline{p^{\prime} u_{i, j}^{\prime}}\right)_{c o m p}$ is required. For this, equation of the fluctuating dilatation $\left(d^{\prime}\right)$ is to be taken into consideration, such equation can be written as

$$
\begin{aligned}
& \frac{d}{d t} d^{\prime}=-2 u_{j, i}^{\prime} \tilde{U}_{i, j}-u_{j, i}^{\prime} u_{i, j}^{\prime}+ \\
& \frac{1}{\bar{\rho}} p_{, i i}^{\prime}+\frac{4}{3} v d_{, i i}^{\prime}+\text { other terms }
\end{aligned}
$$

The other terms are unknown in relation with density fluctuation, mean gradient of density and pressure. We therefore write this equation as follow

$\frac{d}{d t} d^{\prime}=-2 u_{j, i}^{\prime} \tilde{U}_{i, j}+$ other terms

Multiplying both sides of Eq. (25) by $p^{\prime}$ and taking the Reynolds- averaging, we have

$$
\overline{p^{\prime} \frac{d}{d t} d^{\prime}}=-2 \overline{p^{\prime} u_{j, i}^{\prime}} \tilde{U}_{i, j}+\text { other terms }
$$

This equation can be written as follow

$$
\overline{\frac{p^{\prime} d^{\prime}}{\tau}}=-2 \overline{p^{\prime} u_{j, i}^{\prime}} \tilde{U}_{i, j}+\text { other terms }
$$

Where $\tau$ is the characteristic time scale of the dilatation fluctuation (see Taulbee et al.1991), $\tau=\frac{K}{\varepsilon}$ Because $\overline{p^{\prime} d^{\prime}}$ is the trace of $\left(\overline{p^{\prime} u_{i, j}^{\prime}}\right)_{c o m p}$, we can write Eq. (27) as

$$
\frac{\overline{p^{\prime} d^{\prime}}}{\tau}=-2\left(\overline{p^{\prime} u_{j, i}^{\prime}}\right)_{c o m p} \tilde{U}_{i, j}+\text { other terms }
$$

To obtain the final form of $\left(\overline{p^{\prime} u_{i, j}^{\prime}}\right)_{\text {comp }}$ an expression of $\overline{p^{\prime} d^{\prime}}$ is required. It is well known that for this term there are some turbulent models, in this study, we choose those according to the general formulation as $\overline{p^{\prime} d^{\prime}}=A_{i j} \tilde{U}_{i, j}+\ldots \ldots .$.

Where $A_{i j}$ is a second rank tensor.

Sarkar et al. (1991)

$$
\overline{p^{\prime} d}=0,4 M_{t}^{2} \bar{\rho}\left(R_{i j}-\frac{2}{3} K \delta_{i j}\right) \tilde{U}_{i, j}+0,2 \bar{\rho} M_{t}^{2} \varepsilon_{s}
$$

Sarkar (1992)

$$
\begin{aligned}
\overline{p^{\prime} d^{\prime}}= & \alpha_{1} M_{t} \bar{\rho}\left(R_{i j}-\frac{2}{3} K \delta_{i j}\right) \tilde{U}_{i, j}+\alpha_{2} \bar{\rho} M_{t}^{2} \varepsilon_{s} \\
& +\alpha_{3} M_{t}^{2} \bar{\rho} K \tilde{U}_{i, i}
\end{aligned}
$$

The model constants $, \alpha_{1}, \alpha_{2}$ and $\alpha_{3}$ take the values : $\alpha_{1}=0,15, \alpha_{2}=0,2$ and $\alpha_{3}=0,0$
Elbaz and Launder (1993)

$$
\overline{p^{\prime} d^{\prime}}=1,5 M_{t}^{2} \bar{\rho}\left(R_{i j}-\frac{2}{3} K \delta_{i j}\right) \tilde{U}_{i, j}
$$

Ristorcelli (1997)

$$
\overline{p^{\prime} d^{\prime}}=\frac{C_{1} M_{t}}{C_{1}+C_{1} M_{t}^{2}} \bar{\rho}\left(R_{i j}-\frac{2}{3} K \delta_{i j}\right) \tilde{U}_{i, j}
$$

From these models, we can deduce easily the general form of the $A_{i j}$-tensor, namely

$A_{i j}-\frac{1}{3} A_{m m} \delta_{i j}=\bar{\rho} F\left(M_{t}\right)\left(R_{i j}-\frac{2}{3} K \delta_{i j}\right)$

Where $F\left(M_{t}\right)$ is a function of the turbulent Mach number, vanishing in an incompressible flow. As suggested in the above cited models, $F\left(M_{t}\right)$ takes the following expressions:

Sarkar (1991)

$F\left(M_{t}\right)=0,4 M_{t}^{2}$
Sarkar $(1992)$
$F\left(M_{t}\right)=0,15 M_{t}$

Elbaz and Launder (1993)

$F\left(M_{t}\right)=1,5 M_{t}^{2}$

Ristorchelli et al. (1997)

$$
F\left(M_{t}\right)=\frac{C_{1} M_{t}}{C_{1}+C_{2} M_{t}^{2}}
$$

Substituting $\overline{p^{\prime} d^{\prime}}$ by its expression in Eq. (29) and using identification between terms which affect the mean gradient velocity in Eq. (28), we obtain

$$
\left.-2\left(\overline{\left(p^{\prime} u_{i, j}^{\prime}\right.}\right)_{c o m p}-\frac{1}{3} \overline{p^{\prime} d^{\prime}} \delta_{i j}\right)=\frac{1}{\tau}\left(A_{i j}-\frac{1}{3} A_{m m} \delta_{i j}\right)
$$

Using Eq. (34), we deduce for the compressible slow part of the pressure-strain correlation the final form model as follow

$$
\left(\overline{p^{\prime} u_{i, j}^{\prime}}\right)_{\text {comp }}^{*}=\bar{\rho} C^{\prime} F\left(M_{t}\right) \frac{\varepsilon}{K}\left(R_{i j}-\frac{2}{3} K \delta_{i j}\right)
$$

Where $\left(\overline{p^{\prime} u_{i, j}^{\prime}}\right)_{\text {comp }}^{*}=\left(\overline{p^{\prime} u_{i, j}^{\prime}}\right)_{\text {comp }}-\frac{1}{3} \overline{p^{\prime} d^{\prime}} \delta_{i j}$

Regarding the models of $F\left(M_{t}\right)$, we choose the following model

$F\left(M_{t}\right) \propto M_{t}^{2}$

and finally, the deduced model for the term $\left(\overline{p^{\prime} u_{i, j}^{\prime}}\right)_{c o m p}$ is:

$$
\left(\overline{p^{\prime} u_{i, j}^{\prime}}\right)_{c o m p}-\frac{1}{3} \overline{p^{\prime} d^{\prime}} \delta_{i j}=\bar{\rho} \beta M_{t}^{2} \varepsilon b_{i j}
$$

Thus, the proposed extension of the Rotta's model taking account of compressibility can be written as

$$
\phi_{i j}^{*}=-C_{1}\left(1-\beta M_{t}^{2}\right) \bar{\rho} \varepsilon_{s} b_{i j}
$$

\section{Simulation of Compressible MIXING LAYERS}

For the stationary compressible mixing layers, the basic averaged equations are:

$$
\left(\bar{\rho} \tilde{u}_{j}\right)_{, j}=0
$$




$$
\begin{aligned}
&\left(\bar{\rho} \tilde{u}_{i} \tilde{u}_{j}\right)_{, j}=-\left(\bar{\rho} R_{i j}\right)_{, j} \\
&\left(\rho \tilde{u}_{i} \tilde{T}\right)_{, i}=-\pi_{d}+\rho \varepsilon-\left(\overline{\rho u_{i}^{\prime \prime}} T^{\prime \prime}\right)_{, i} \\
&\left(\bar{\rho} \tilde{u}_{k} R_{i j}\right)_{, k}= P_{i j}+\phi_{i j}^{*}+\left(\overline{\rho u_{i}^{\prime \prime} u_{j}^{\prime \prime} u_{k}^{\prime \prime}}\right)_{, k} \\
&-\frac{2}{3} \varepsilon \delta_{i j}+\frac{2}{3} \pi_{d} \delta_{i j} \\
& \phi_{i j}^{*}=\phi_{i j}-\frac{2}{3} \pi_{d}
\end{aligned}
$$

The gradient diffusion hypothesis is used to model:

-The turbulent heat flux

$$
\left(\overline{\rho u_{k}^{\prime \prime} T}\right)=-C_{T} \frac{K}{\varepsilon} R_{k m} \tilde{T}_{, k}
$$

-The diffusion term

$$
D_{i j}=\left(C_{s} \frac{K}{\varepsilon} \overline{\rho u_{i}^{\prime \prime} u_{j, m}^{\prime \prime}}\right)_{, m}
$$

Classically, the second order closure suggests to determine the dissipation term $\boldsymbol{E}_{i j}$ by using isotropic dissipation model:

$\varepsilon_{i j}=\frac{2}{3} \varepsilon \delta_{i j}$

Recently, a concept of the dissipation was proposed by Sarkar (1991) :

$$
\begin{aligned}
& \varepsilon=\varepsilon_{s}+\varepsilon_{c} \\
& \varepsilon_{s}=v \overline{\omega_{i} \omega_{i}}, \quad \varepsilon_{c}=\frac{4}{3} v \overline{d^{\prime 2}}
\end{aligned}
$$

The dissipation solenoidal $\varepsilon_{s}$ can be modelled by using the traditional model, namely

$$
\begin{aligned}
\frac{\partial}{\partial t}\left(\bar{\rho} \varepsilon_{s}\right)+\left(\bar{\rho} \tilde{U}_{m} \varepsilon_{s}\right)_{, m}= & \frac{\varepsilon_{S}}{k}\left(C_{\varepsilon 1} R_{m n} \tilde{U}_{m, n}-\bar{\rho} C_{\varepsilon 2} \varepsilon_{s}\right) \\
& +\left(C_{\varepsilon 3} \frac{k}{\varepsilon} R_{m n} \varepsilon_{s, n}\right)_{, m}
\end{aligned}
$$

In this work, the model of Sarkar is used to express the pressure dilatation correlation and the turbulent dilatation of dissipation:

$$
\varepsilon_{c}=0,5 \varepsilon_{s} M_{t}^{2}
$$

For the model of Adumitroiae et al.(1999), the compressible parameters $d_{1}$ and $d_{2}$ are determined by using the model of Sarkar (1992)

$d_{2}=0.15 M_{t}$ and $d_{1}=0$.

\section{RESUlts AND DisCUSSION}

The two free streams of the fully developed compressible mixing layer are characterized typically by the convective Mach number $M_{C}$, the parameters

$s=\frac{\rho_{2}}{\rho_{1}}$ and $r=\frac{U_{2}}{U_{1}}$ are respectively the density and velocity ratios, the experiments conditions of Goebel et al.(1991) are listed in Table.1

The values of the constants models used in the present simulation are:

$C_{\varepsilon 1}=1,4, C_{\varepsilon 2}=1,8, C_{\mu}=0,09, C_{\varepsilon}=0,25, C_{T}=0,26$.

In this study, we consider predictions of two models for the pressure-strain correlation: the Launder Reece and Rodi (LRR) model(1975) and the Adumitroiae et al. model (1999). Two versions of the Adumitroiae et al. model will be considered : the original version (without correction of the $C_{1}$-coefficient and the version where the proposed $M_{t}$-correction of $C_{1}$ is included.

Table 1 Experience of Goebel and Dutton

\begin{tabular}{|c|c|c|c|c|c|}
\hline$M C$ & 0.2 & 0.46 & 0.69 & 0.86 & 1 \\
\hline$r=\frac{U_{2}}{U_{1}}$ & 0.78 & 0.57 & 0.18 & 0.16 & 0.16 \\
\hline$s=\frac{\rho 2}{\rho 1}$ & 0.76 & 1.55 & 0.57 & 0.6 & 1.14 \\
\hline
\end{tabular}

The fundamental parameter characterizing the effects of compressibility on the mixing layer is the growth rate $\frac{d \delta}{d x}, \delta$ denotes the momentum thickness of the mixing layer. Figure 1 shows the comparison between the computed normalized growth rate by its incompressible counterpart $G=\frac{d \delta}{d x} /\left(\frac{d \delta}{d x}\right)_{M_{c}=0}$ with different experiment results available in the literature and with those obtained by empirical formula of Dimotakis (1991) :

$$
G=0,8 \exp \left(-M_{t}^{2}\right)+0,2
$$

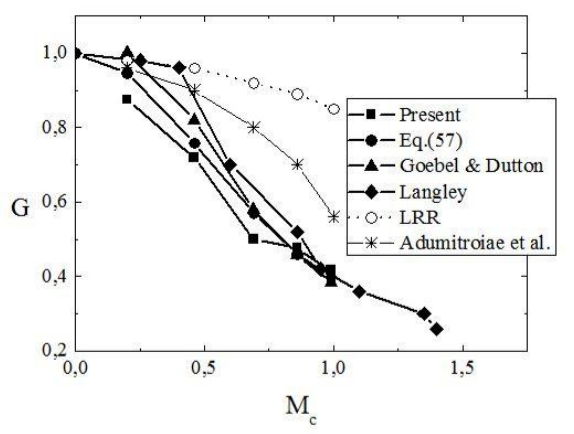

Fig.1. Normalized growth rate $\mathrm{G}$ versus $M_{c}$

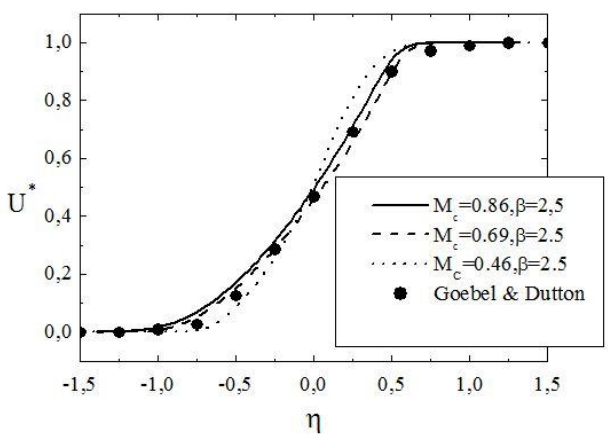

Fig. 2. Similarity of the mean velocity

The calculated growth rate $\mathrm{G}$ decreases with increasing convective Mach number: a phenomenon which has often been observed in experimental studies of compressible mixing layers. The LRR model over predicts the growth rate $G$ and similar results are obtained for the Reynolds stress similarity (results are not included in Fig. 2 and Fig.3). With the proposed correction of the slow part of the pressure-strain 
correlation, the model of Adumitroiae et al. is now able to properly predict the growth rate $\mathrm{G}$.

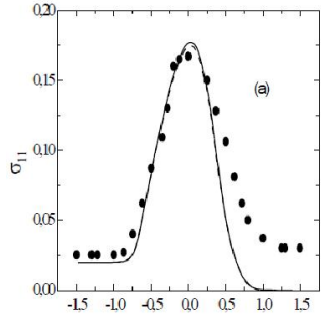

$\eta$
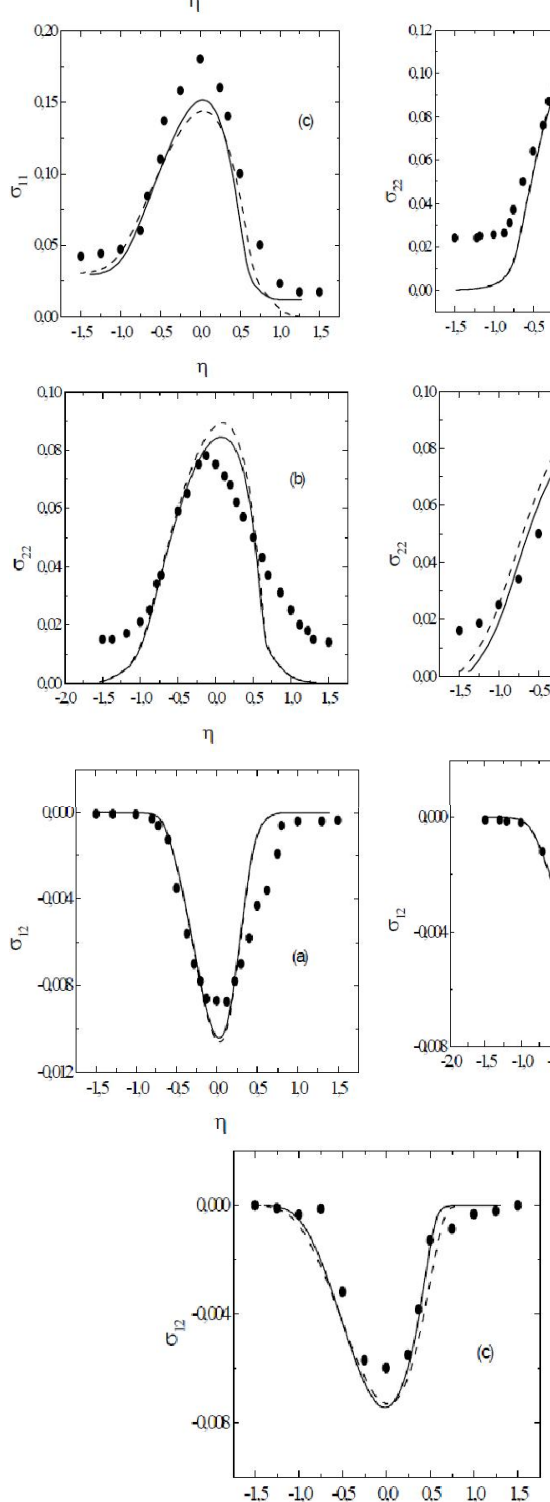
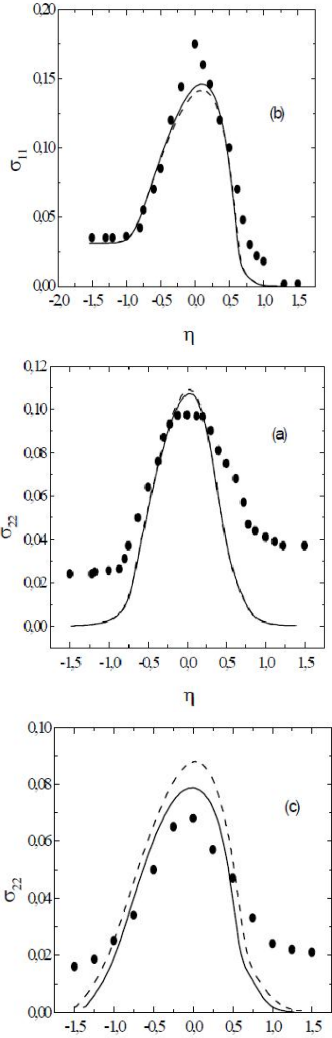

$\eta$

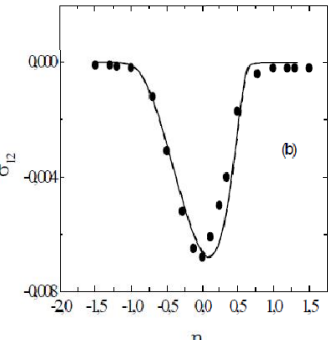

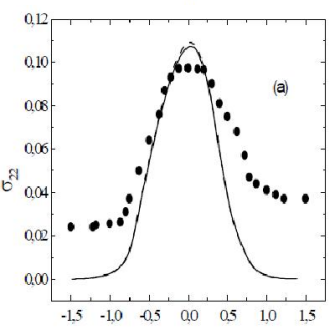

stream coordinate and $y_{c}$ is the cross-stream coordinates corresponding to $U^{*}=0,5$.

The calculated velocity profiles with the corrected version of the Adumitroiae et al. model are in reasonable agreement with experimental data. Figure 3 shows the computed results of the Reynolds similarity intensities: the streamwise intensity $\left.\sigma_{11}=\sqrt{R_{11} /\left(U_{1}-U_{2}\right.}\right)^{2}$, the transverse intensity $\sigma_{22}=\sqrt{R_{22} /\left(U_{1}-U_{2}\right)^{2}}$, and the shear stress $\sigma_{12}=R_{12} /\left(U_{1}-U_{2}\right)^{2}$, obtained from the two versions model of Adumitroiae et al. are compared with experiments results of Goebel and Dutton. It is clear that the two versions model lead to similar results for small value of convective Mach number $\left(M_{C}=0,46\right)$. But, when the compressibility effects are more significant $\left(M_{C}=0,69, M_{C}=0,86\right)$, the effects of the proposed correction model are clearly manifested on the normal turbulent Reynolds stress, particularly on the transverse similarity intensity. But the shear stress is not affected by the proposed correction model.

\section{Conclusion}

In this study, the widely used second order closures has been used for the prediction of compressible mixing layer. The standard -stress closure with the addition of the dilatational terms: the pressure dilatation correlation and the turbulent dissipation of the dilatation yields very poor predictions of the changes in the Reynolds stress anisotropy magnitude. The deficiencies of this closure is due to the use of the incompressible models of the pressure-strain correlation. This term controls the structural compressibility effects on the turbulence.

A modification of the standard Rotta's model of the slow part of the pressure strain correlation has been proposed. The usual coefficient $C_{1}$ becomes dependent on the turbulent Mach number $M_{t}$. In general, the proposed compressibility model with the Adumitroiae model of the mean part of the pressure strain successfully predict the reduced growth rate and the decrease of the normal Reynolds stress peaks with increasing convective Mach number. Also the similarity velocity is well predicted by the proposed model. This leads to the conclusion that the compressibility correction of the slow part of the pressure strain correlation model is found out to be an important issue in the second order closure for the compressible turbulent flows.

\section{ACKNOWLEDGEMENTS}

This work is realized in the Fluid Mechanics Laboratory, F.S.T, Tunis, Tunisia.

\section{REFERENCES}

Adumitroiae, V., J.R. Ristorchelli and D.B. Taulbee(1999). Progress in Favre Reynolds stress closure . Physics of Fluids A(9), 2696-2719. 
H. Khlifi and T. Lili / JAFM, Vol. 4, No. 2, Special Issue, pp. 99-104, 2011.

Dimotakis, P.E.(1991). Turbulent free shear layer mixing and combustion. In Murhy and curran (eds) progress in astronautics and aeronautics. AIAA, Washington DC. 137.

Elliot, G.S. and M. Samimy (1990). Effects of compressible effects in free shear layers. Physics of Fluids $\mathrm{A}(2), 1231$.

El-Baz, A.M. and B.E Launder (1993). Second moment modeling of compressible mixing layers. In W. Rodi and F.Martelli, editors, Engineering Turbulence Modeling and Experiments 2, 63-72.

Favre, A.(1965). Statistical equations of turbulent gases. Jour. Mecan. 4, 361-390.

Goebel , S.G., J.C. Dutton, H. Krier and J.P. Rence(1990). Mean and turbulent velocity measurements of supersonic mixing layers. Experiments in Fluids 8, 263-272.

Launder, B.E., G.J. Reece and Rodi (1975). Progress in the development of a Reynolds stress turbulence closure. Journal of Fluid Mech. 68, 537-566.

Ristorcelli, J.R. (1977). A pseudo-sound constitutive relationship for the dilatational covariance in compressible turbulence. Journal of Fluid Mech. 347, 37-70.

Sarkar, S., Erlebacher, G., M.Y. Hussaini, and H.O. Kress, (1991). The analysis and modeling of dilatational terms in compressible turbulence. Journal of Fluid Mech. 277, 473-493.

Sarkar, S. (1992). The pressure dilatation correlation in compressible flows. Physics of Fluids A(4), 26742682.

Sarkar, S. (1995). The stabilizing effects of compressibility in turbulent shear flows. pressure dilatation correlation in compressible flows. Journal of Fluid Mech. 282, 163-186.

Taulbee , D. and J. Van Osdol(1991). Modeling turbulent compressible flows. The mass fluctuating velocity and squared density. AIAA paper 91-0524.

Rotta,J.(1951). Statiche theorie nichthomogener . Turbulenz Zeitschrift für Physik 129, 547-572. 